\title{
Magnetoresistive effects in planar NiFe nanoconstrictions
}

\author{
S. H. Florez, M. Dreyer, K. Schwab, C. Sanchez, and R. D. Gomez \\ Laboratory for Physical Sciences, College Park, Maryland and Department of ECE, \\ University of Maryland, College Park, Maryland 20742
}

(Presented on 6 January 2004)

\begin{abstract}
This study focuses on domain wall resistance in $\mathrm{Ni}_{80} \mathrm{Fe}_{20}$ nanowires containing narrow constrictions down to $15 \mathrm{~nm}$ in width. Distinct differences in the magnetoresistance curves were found to depend on the constriction size. Wider constrictions are dominated by the overall anisotropic magnetoresistance of the structure, while constrictions narrower than $\sim 40 \mathrm{~nm}$ exhibit an additional distinct contribution from a domain wall. The effect is negative and typically varies from $1 \%$ to $5 \%$.

(C) 2004 American Institute of Physics. [DOI: 10.1063/1.1682831]
\end{abstract}

\section{INTRODUCTION}

It is now recognized that the domain walls formed in magnetic structures contribute to the magnetoresistance (MR). This has been observed through many experiments and numerous theoretical formulations ${ }^{1-5}$ have been proposed. Despite these efforts, no unified theoretical picture exists and inconsistencies ${ }^{6-9,11}$ within reported results remain unresolved. This is partly due to the difficulty in isolating a single domain wall and distinguishing its contribution from other effects such as the anisotropic magnetoresistance of the magnetic domains. In this work, we fabricated structures designed to trap a single domain wall. We focused on NiFe because of its technological importance and the controversy that remains concerning the sign of the domain wall resistance effect. ${ }^{6-8}$ Specifically, we performed MR measurements and magnetic force microscopy imaging on nanowires that contain localized constrictions. The purpose of the constriction is twofold. First, to magnetically decouple the wires, and second, to act as a pinning center for domain walls.

\section{EXPERIMENT}

The nanostructures were fabricated on oxidized Si substrates through $e$-beam lithography using the standard lift-off method. These consist of two NiFe wires, roughly $200 \mathrm{~nm}$ wide, $25 \mathrm{~nm}$ thick and joined by narrow constrictions from $15-100 \mathrm{~nm}$. Fine control of junction widths (nanometers precision) was achieved by slightly varying the $e$-beam exposure dose along an array of identical structures. The metals were deposited using thermal evaporation at background pressures near $1 \times 10^{-6} \mathrm{mbar}$. The wires were evaporated from a $\mathrm{Ni}_{81} \mathrm{Fe}_{19}$ source to obtain a nominal $\mathrm{Ni}_{80} \mathrm{Fe}_{20}$ composition of the sample. Figure 1(a) shows a series of the structures and the $\mathrm{Au} / \mathrm{Cr}$ electrical contacts. The small rectangular pads, visible in Fig. 1(a), (see arrow) are additional Au pads and not to be confused with nucleation pads for the NiFe wire. These pads are only $\sim 25 \mathrm{~nm}$ thick, while the large $\mathrm{Au} / \mathrm{Cr}$ contact structures are $>100 \mathrm{~nm}$ thick and suitable for wire bonding. The difference in the lengths of the elements, the angle between them $\left(\sim 27^{\circ}\right)$, and the size of the constriction were features designed to magnetically decouple the wires and separate their switching fields. Figures 1(b) and 1(c), at higher magnification, show examples of some of the fabricated junctions.

The high aspect ratio of the wires promotes single domain states for both elements, as confirmed by MFM images taken at remanence and shown in Fig. 2. The bright and dark contrast at the ends and at the junction, correspond to the accumulation of magnetic charges in those regions. From the contrast one can infer that the magnetizations of the elements are connected head to tail and follow the easy axes of the respective elements.

\section{RESULTS AND DISCUSSION}

A representative magnetoresistance curve of structures with wide junctions $(\sim 100 \mathrm{~nm})$ is shown in Fig. 3. The mag-
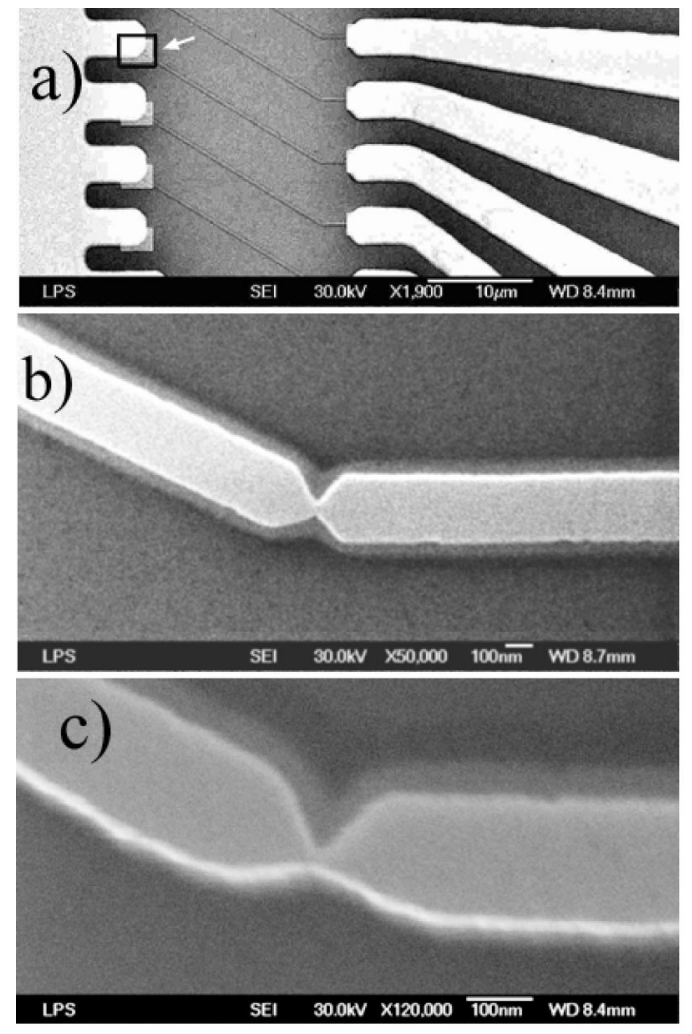

FIG. 1. SEM images of junctions at several magnifications $(a) \times 1900$, (b) $\times 50000$ and $(\mathrm{c}) \times 120000$. 


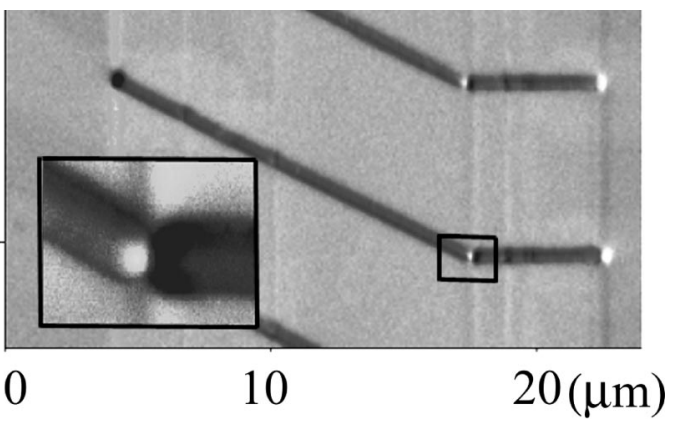

FIG. 2. MFM images of NiFe wires.

netic field was directed parallel to the axis of the short element. The MR exhibits the familiar nonlinear behavior and is accompanied by an abrupt upward transition at a magnetic field value $H=H_{\mathrm{sw} 2}$. The behavior is exclusively governed by the long element, since the magnetization of the short element is always parallel to the current and consequently yields negligible AMR contribution. Starting from near negative saturation the resistance increases as the magnetization of the long element becomes increasingly parallel to its easy axis, i.e., the direction of the current flow. At positive fields, the MR curve decreases more rapidly as the average magnetization rotates away from the easy axis. As the field is further increased, the magnetization switches direction at $H$ $=H_{\mathrm{sw} 2}$. Since this element forms a $27^{\circ}$ angle with the applied field, the magnetization after the switch will make a lesser angle with the easy axis than before. Hence, it will have less transverse component with respect to the current direction and thus produce higher resistance at $H=H_{\mathrm{sw} 2}$. Furthermore, by virtue of strong exchange and dipolar interaction resulting from the large junction area, the short element switches simultaneously (at the given field sweeping rate) and the intermediate state, where opposing magnetizations are created at the junction, is suppressed. The behavior is identical in the return phase of the MR loop.

The situation is markedly different when the junction size is reduced below $\sim 50 \mathrm{~nm}$. An example of this case is shown in Fig. 4. The main distinction is the appearance of an abrupt resistance drop at $H=H_{\text {sw } 1}$ followed by a gradual decrease reminiscent of the earlier case at negative fields. The low resistance state persists within a finite field range up

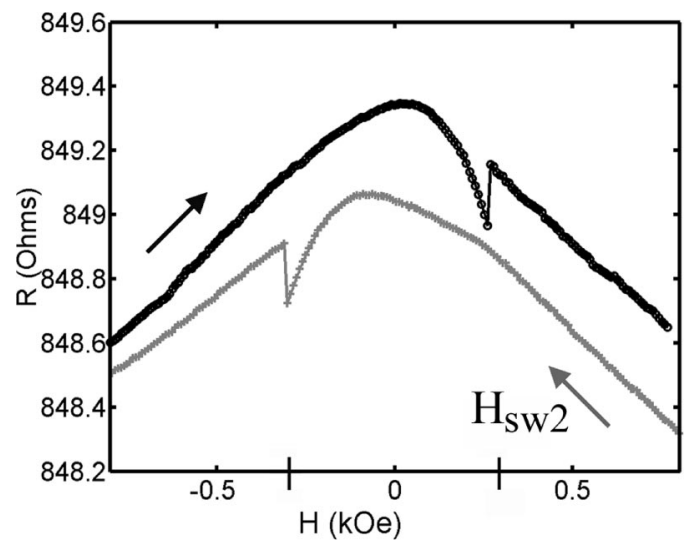

FIG. 3. MR response for an element with wide junction $>60 \mathrm{~nm}$.

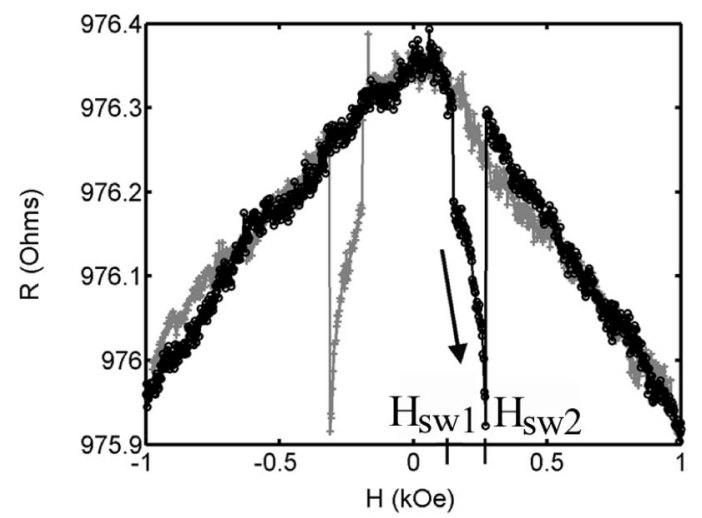

FIG. 4. MR response for an element with an $\sim 15 \mathrm{~nm}$ constriction.

to $H=H_{\mathrm{sw} 2}$. The resistance jumps were found to be irreversible, and supporting data are shown in Fig. 5(a). The top curve is a segment of a half cycle MR curve, which shows both negative $\left(H_{\mathrm{sw} 1}\right)$ and positive $\left(H_{\mathrm{sw} 2}\right)$ transitions with increasing field. In the bottom curves the magnetic field was swept from negative to positive values until the sharp drop in resistance was observed at $H=H_{\mathrm{sw} 1}$. Then, prior to reaching $H=H_{\mathrm{sw} 2}$, the field was reduced and swept in the opposite direction, as denoted by the arrows in the figure. The low resistance state persisted past $H=0$, and a positive transition was observed at a negative field $H=H^{\prime}$ as shown. This is characteristic of an irreversible process due to a domain wall, and strongly suggestive that $H=H^{\prime}$ is the field at which the domain wall is swept out of the junction accompanying the reversal of the small element.

Additional insight on the low resistance state can be derived by considering the MFM images shown in Fig. 5(b), which show a small area enclosing the short element in its
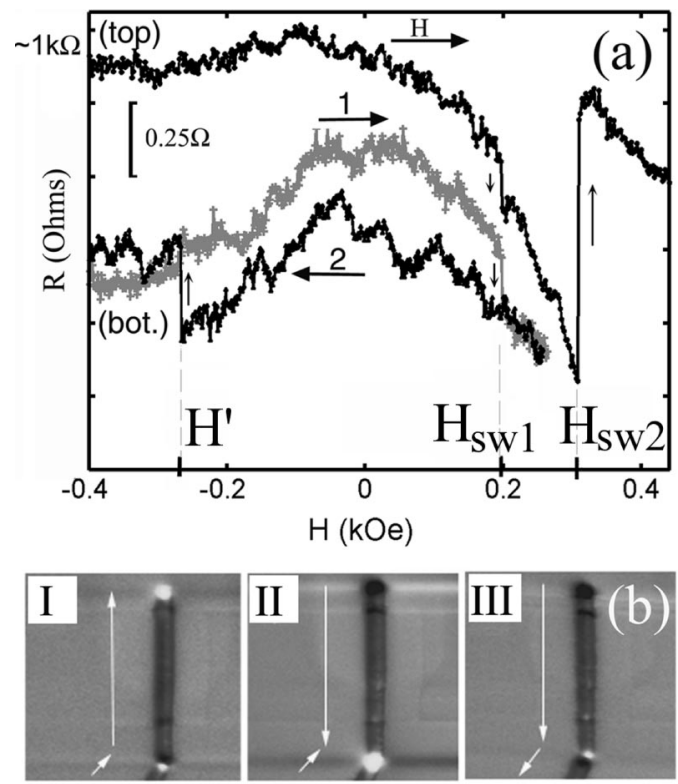

FIG. 5. (a). The top curve is a segment of a half cycle MR curve. For the bottom curves, the field is increased (1) until the resistance drop was observed and then decreased (2), while still in the low resistance state. A positive resistance jump is not observed until a positive field $\mathrm{H}^{\prime}$. (b). MFM images of three observed magnetic states. 
entirety. These images were obtained in the presence of external magnetic fields parallel to the short element [vertical axis in Fig. 5(b)]. The constriction is located in the lower part of the image where we observe the effects of magnetic charge accumulation. While the MFM resolution is insufficient to observe the DW structure at the junction, it is nevertheless clear that the orientation of the net magnetization of the elements can be deduced as shown by the arrows. In this case, i.e., patterns with narrow constrictions, three stable magnetic states were identified. We submit that the low resistance state bounded by $H_{\mathrm{sw} 2}$ and $H_{\mathrm{sw} 1}$ coincides with image (II) in Fig. 5(b). It shows a strong positive charge accumulation characteristic of head to head magnetizations. The correlation is made from independent MR and MFM measurements on the same structure. Although it would have been ideal to obtain MR and MFM simultaneously, this was not possible since the field from the MFM tip perturbed the local magnetization which introduced large fluctuations in the MR during the scan. Indeed we found, through MFM scanning in the presence of applied external field, that switching occurred earlier than for the MR measurement. This was undoubtedly due to the additional field produced by the MFM tip.

Based on these observations, we suggest that the abrupt reduction of the resistance at $H_{\mathrm{sw} 2}$ occurred due to the formation of a DW at the junction. The abrupt resistance rise at $H_{\text {sw1 }}$ is caused by the subsequent switching of the longer element, as shown in right panel of Fig. 5(b). Although the values of $H_{\mathrm{sw} 1,2}$ depend on the sweep rate and on the value of the current, consistent with a domain wall processes, narrower constrictions seem to yield wider field ranges $\Delta H$ $=H_{\mathrm{sw} 1}-H_{\mathrm{sw} 2}$. This further supports our description since width reduction decouples the wires by reducing the exchange energy at the constriction. However, without enough statistical correlations it is hard to rule out the influence of other factors such as intrinsic switching field distributions and slight variations in the widths of element.

From the data, we can calculate the effective resistivity contribution from a single domain wall. Supposing that the resistivity in the junction changes from $\rho$ to $\rho+\Delta \rho$, due to the presence of a DW then, the total resistance with and without the DW are, respectively, $R^{\mathrm{DW}}=\rho L / t w+(\rho$ $+\Delta \rho) \delta_{w} / t w_{\mathrm{j}}$ and $R=\rho L / t w+\rho \delta_{w} / t w_{\mathrm{j}}$. Here, $L$ is the total length, $t$ the thickness $(t \sim 25 \mathrm{~nm}), w$ the width of the wire $(w \sim 200 \mathrm{~nm}), w_{j}$ the width of the junction, and $\delta_{w}$ the DW width. Assuming $w_{j} \sim \delta_{w}$ (Ref. 10) and $L / w \sim 100$, the normalized change in resistance is given by $\left(R^{\mathrm{DW}}-R\right) / R$ $=(\Delta \rho / \rho) /(L / w+1)$. For the data shown in Fig. 4 , changes in resistance are roughly $\Delta R / R \sim 0.03 \%$, resulting in negative $\Delta \rho / \rho \%$ in the DW near 3\%. Larger changes up to $5 \%$ were also observed. This sharp negative drop in resistance is most likely due to the AMR effect originating from within the domain wall trapped at the junction. However, several other mechanisms where domain walls cause a decrease in resistance have also been proposed. ${ }^{3-5}$

We would be remiss, however, if we did not mention that for one specific sample, a positive domain wall resistance was observed with similar features. This raises doubts concerning AMR as the exclusive origin of the observed domain wall resistance. It appears that the situation involves subtle differences in preparation. Ultimately, the sign of the DW resistance in our $\mathrm{NiFe}$ wires may therefore be affected by slight stoichiometry, morphological variations and by the type of impurities present. This may point toward van Gorkom et al. ${ }^{3}$ semiclassical model for DWR, for which the effect may have either sign, depending on the ratio of spin scattering lifetimes $\tau_{ \pm}$. Following their analysis for the case of bulk NiFe with $\tau_{+}>\tau_{-}$, leads to a positive effect. However, as pointed out by Theeuwen et al., ${ }^{8}$ this ratio my change sign within a constriction as a consequence of spin dependent surface scattering of electrons within the DW.

\section{ACKNOWLEDGMENT}

This work was supported by NSF ECS Grant No. 9984797.

${ }^{1}$ G. G. Cabrera and L. M. Falicov, Phys. Status Solidi B 62, 217 (1974).

${ }^{2}$ P. M. Levy and S. Zhang, Phys. Rev. Lett. 79, 5110 (1997).

${ }^{3}$ R. P. van Gorkom, A. Brataas, and G. E. W. Bauer, Phys. Rev. Lett. 83, 4401 (1999)

${ }^{4}$ G. Tatara and H. Fukuyama, J. Magn. Magn. Mater. 177-181, 193 (1998).

${ }^{5}$ A. D. Kent et al., IEEE Trans. Magn. 34, 900 (1998).

${ }^{6}$ Y. B. Xu et al., Phys. Rev. B 46, R014901 (2000).

${ }^{7}$ C. Yu et al., J. Appl. Phys. 93, 8761 (2003).

${ }^{8}$ S. J. C. H. Theeuwen et al., J. Appl. Phys. 89, 4442 (2000).

${ }^{9}$ Y. Otani et al., IEEE Trans. Magn. 34, 1096 (1998).

${ }^{10}$ P. Bruno, Phys. Rev. Lett. 83, 2425 (1999).

${ }^{11}$ U. Ebels et al., Phys. Rev. Lett. 84, 983 (2000). 